\title{
A combined Cox and logistic model provides accurate predictive performance in estimation of time-dependent probabilities for recurrence of intrahepatic cholangiocarcinoma after resection
}

\author{
Seogsong Jeong ${ }^{1,2 \#}$, Guijuan Luo ${ }^{2,3 \#}$, Qiang Gao ${ }^{4 \#}$, Jing Chen ${ }^{2,3 \#}$, Xiaolong Liu ${ }^{5}$, Liangqing Dong ${ }^{4}$, \\ Yongjie Zhang ${ }^{6}$, Feng Shen ${ }^{7}$, Qingbao Cheng ${ }^{8}$, Chengjun Sui ${ }^{9}$, Jingfeng Liu ${ }^{5}$, Hongyang Wang ${ }^{2,3}$, \\ Qiang Xia ${ }^{1}$, Lei Chen ${ }^{2,10}$
}

${ }^{1}$ Department of Liver Surgery, Renji Hospital, School of Medicine, Shanghai Jiao Tong University, Shanghai, China; ${ }^{2}$ International Cooperation Laboratory on Signal Transduction, Eastern Hepatobiliary Surgery Institute, Second Military Medical University, Shanghai, China; ${ }^{3}$ National Center for Liver Cancer, Shanghai, China; ${ }^{4}$ Department of Liver Surgery and Transplantation, Liver Cancer Institute, Zhongshan Hospital, and Key Laboratory of Carcinogenesis and Cancer Invasion (Ministry of Education), Fudan University, Shanghai, China; ${ }^{5}$ Mengchao Hepatobiliary Hospital, Fujian Medical University, Fuzhou, China; ${ }^{6}$ Biliary Tract Department II, Eastern Hepatobiliary Surgery Hospital, Second Military Medical University, Shanghai, China; ${ }^{7}$ Department of Hepatobiliary Surgery, Eastern Hepatobiliary Surgery Hospital, Second Military Medical University, Shanghai, China; ${ }^{8}$ Biliary Tract Department I, Eastern Hepatobiliary Surgery Hospital, Second Military Medical University, Shanghai, China; ${ }^{9}$ Department of Special Medical Care \& Liver Transplantation, Eastern Hepatobiliary Surgery Hospital, Second Military Medical University, Shanghai, China; ${ }^{10}$ Shanghai Cancer Center, Shanghai Medical College, Fudan University; Shanghai, China

Contributions: (I) Conception and design: S Jeong, G Luo, Q Gao, J Chen, J Liu, H Wang, Q Xia, L Chen; (II) Administrative support: All authors; (III) Provision of study materials or patients: S Jeong, G Luo, Q Gao, J Chen, J Liu, H Wang, Q Xia, L Chen; (IV) Collection and assembly of data: All authors; (V) Data analysis and interpretation: S Jeong, G Luo, Q Gao, J Chen, J Liu, H Wang, Q Xia, L Chen; (VI) Manuscript writing: All authors; (VII) Final approval of manuscript: All authors.

\#These authors contributed equally to this work.

Correspondence to: Lei Chen; Hongyang Wang. International Cooperation Laboratory on Signal Transduction, Eastern Hepatobiliary Surgery Institute, Second Military Medical University, 225 Changhai Road, Shanghai 200438, China. Email: chenlei@smmu.edu.cn; hywangk@vip.sina.com; Qiang Xia. Department of Liver Surgery, Renji Hospital, School of Medicine, Shanghai Jiao Tong University, 160 Pujian Road, Shanghai 200127, China. Email: xiaqiang@shsmu.edu.cn.

Background: Intrahepatic cholangiocarcinoma has heterogeneous outcomes after resection. There remains a need for broadly applicable recurrence-specific tool offering precise evaluation on curativeness of resection.

Methods: A four hospital-based clinical cohort involving 1,655 patients with intrahepatic cholangiocarcinoma who received surgical resection were studied. Cox and logistic models were networked into one system containing risk categories with distinctive probabilities of recurrence. Prediction of timeto-recurrence was performed by formulizing time-dependent risk probabilities. The model was validated in three clinical cohorts $(n=332)$.

Results: From the training cohort, 10 and 11 covariates, including diabetes, cholelithiasis, albumin, platelet count, alpha fetoprotein, carbohydrate antigen 19-9, carcinoembryonic antigen, hepatitis B virus infection, tumor size and number, resection type, and lymph node metastasis, from Cox and logistic models were identified significant for recurrence-free survival (RFS). The combined Cox \& logistic ranking system (CCLRS)-adjusted time-dependent probabilities were categorized into seven ranks (5-yr RFS for lowest and highest ranks were $75 \%$ vs. $0 \%$; hazard ratio 18.5 , 95\% CI: 14.7-24.9, $\mathrm{P}<0.0001)$. The CCLRS was validated with a minimum area under curve value of 0.8086 . Prediction of time-to-recurrence was validated to be excellent (Pearson r, 0.8204; $\mathrm{P}<0.0001$ ).

Conclusions: The CCLRS allows precise estimation on risk of recurrence for intrahepatic cholangiocarcinoma after resection. It could be applicative when estimating time-dependent disease status and stratifying individuals who sole resection of the tumor would not be curative. 
Keywords: Primary liver cancer; biliary malignancy; hepatectomy; surgery; resection; regression model; nomogram.

Submitted Aug 23, 2019. Accepted for publication Dec 21, 2019.

doi: 10.21037/hbsn.2020.01.07

View this article at: http://dx.doi.org/10.21037/hbsn.2020.01.07

\section{Introduction}

Intrahepatic cholangiocarcinoma (ICC) is the second common primary hepatic malignancy after hepatocellular carcinoma accounting for $10 \%$ to $20 \%$ of all primary liver cancers (1). The incidence and mortality of ICC is increasing in both Eastern and Western countries over 3 decades $(2,3)$. Resection is the standard of care for resectable ICC that provides 5 -year overall survival rates of $20 \%$ to $40 \%$ (4).

Recent staging systems for survival prediction in patients with ICC have highlighted the applicability of the Cox hazard proportional regression model (5) to generate predictive signature to reflect time-dependent risk, which were visualized as a nomogram to improve accessibility (6-9). However, these systems failed in achieving high accuracy in ICC (C-index $<0.7)$. For clinicians, a novel predictive tool with high discriminative capacity is demanded to identify populations with ICC under high risk of recurrence after surgical resection to consider other treatment modalities or combine with other treatments rather than surgical resection only and to manage individualized follow-up schedules. For patients, timedependent risk of recurrence is demanded. However, current methodologies and staging systems are ineligible in performing precise estimation of recurrence after resection (10).

Herein, we sought to develop a model to address clinical concerns regarding ICC after resection that there is no supportive tool with high precision for estimation of postresection time-dependent risk probability of recurrence. We present the following article in accordance with the TRIPOD reporting checklist (available at https://hbsn. amegroups.com/article/view/10.21037/hbsn.2020.01.07/rc).

\section{Methods}

\section{Study patients}

This study is a retrospective multicenter clinical cohort study based on a dataset of patients with ICC who underwent surgical resection and met the inclusion criteria between 2008 and 2015 at 4 hospitals, including Eastern Hepatobiliary Surgery Hospital (Shanghai, $\mathrm{CN} ; \mathrm{n}=1,477$ ), Renji Hospital (Shanghai, CN; n=106),
Mengchao Hepatobiliary Hospital (Fuzhou, CN; n=14), and Zhongshan Hospital (Shanghai, CN; $n=246$ ). Due to incomplete clinical and/or follow-up data, 154, 22, and 12 patients from Eastern Hepatobiliary Surgery Hospital, Renji Hospital, and Zhongshan Hospital were excluded from the present study, respectively. The model was derived from 1,323 patients from the Eastern Hepatobiliary Surgery Hospital, and validated in three other clinical cohorts, including Renji Hospital; validation cohort 1 $(\mathrm{n}=84)$, Mengchao Hepatobiliary Hospital; validation cohort 2 ( $\mathrm{n}=14)$, and Zhongshan Hospital; validation cohort $3(\mathrm{n}=234)$. This study was performed in accordance with the Declaration of Helsinki (as revised in 2013) and the transparent reporting of a multivariable prediction model for individual prognosis or diagnosis (TRIPOD) statement (11). Informed consents were obtained, and the ethics approval was waived by the local institutional review board according to the retrospective nature of the study.

\section{Inclusion criteria and definition of ICC}

All patients were screened for the following inclusion criteria: (I) surgical resection as initial treatment, (II) pathologic confirmation of ICC, (III) no perihilar cholangiocarcinoma, (IV) no distant metastasis, (V) no lymph node metastasis except for hepatoduodenal ligament, retropancreatic, and paraaortic lymph node metastases, and (VI) no perioperative death (deceased within 1 month after surgery). All ICC were confirmed pathologically when CK7, CK19, and MUC1 were positive, and CK20, HepPar1, and glypican-3 were negative (12).

\section{Data collection and resection}

Tumor-related data, including tumor diameter, number, vascular invasion, and lymph node metastasis were obtained from pathology. Surgical resection was performed as described previously (8). Minor resection/hepatectomy is defined as a partial hepatectomy with a resected extent of less than hemihepatectomy. Hepatoduodenal ligament, retropancreatic, and paraaortic lymph nodes were routinely dissected. 


\section{Follow-up}

All patients were regularly followed and monitored for recurrence by computed tomography or magnetic resonance imaging one month after surgery, once every 3 months up to second year, once every 6 months until third year, and twice every year thereafter. Recurrence was confirmed when a lesion was proven by biopsy or deemed suspicious on imaging studies in the setting of CA19-9 elevation. Median follow-up of the training cohort was 50.3 months [interquartile range (IQR), 35.6 to 68.9 months]. There was no patient with recurrence after 5 years of follow-up, thus recurrence after 5 years was not included in the model as event.

\section{Model derivation}

Risk probabilities of postresection recurrence estimated from Cox and logistic multivariable regression models were calculated in accordance with the following formulas:

$$
\begin{aligned}
& \operatorname{Cox}_{\text {Probability }}=\frac{\theta i}{\sum_{j: y j y i} \theta j} \\
& \text { Logistic }_{\text {Probability }}=\frac{1}{1+e^{-a}}
\end{aligned}
$$

Event observed to have occurred with subject $i$ at time $Y$ and $\theta_{j}=\exp \left(X_{j} \cdot \beta\right)$. The summation is over the set of subjects $j$ where the event has not occurred before time $Y i$, including subject itself. For logistic probability, $a$ was $w_{0}+w_{1} x_{1}+\cdots+w_{\mathrm{n}} x_{\mathrm{n}}$, when $w$ is the coefficient and $x$ is input of the covariate. The probabilities for Cox and logistic models were adjusted into one probability for the CCLRS using square-dependent ranks and optimum thresholds obtained from receiver operating characteristic (ROC) curves.

\section{Statistical analysis}

The primary endpoint was recurrence of ICC after surgical resection, including both intrahepatic and extrahepatic detection by imaging studies, defined as time from surgery to recurrence; recurrence-free survival. All demographic and clinical characteristics were tested for association with recurrence-free survival in the training cohort using Cox proportional hazards model and logistic regression. Regarding relatively long-term follow-up periods along with proportion of recurrence cases before the median followup $(98.2 \%)$, censored subjects were also included for the logistic regression. Demographic and clinical characteristics were presented with median and IQR or categorized according to general cut-off values. For evaluation of the performance accuracy and discriminative capacity, $\mathrm{R}^{2}, \mathrm{~g}$, and gr indices, which were achieved from the $\mathrm{R}$ project, and brier score (BS; $\sum\left(e_{\mathrm{i}}-o_{\mathrm{i}}\right) / \mathrm{n} ; e=$ estimate; $o=$ observation [0 or 1]) and Dxy (Somers' D; $\mathrm{N}_{C}-\mathrm{N}_{\mathrm{D}} / \mathrm{N}_{\mathrm{C}}+\mathrm{N}_{\mathrm{D}}+\mathrm{N}_{\mathrm{T}} ; \mathrm{N}_{\mathrm{C}}$ = concordant pairs; $\mathrm{N}_{\mathrm{D}}=$ discordant pairs; $\mathrm{N}_{\mathrm{T}}=$ neither concordant nor discordant pairs) were employed (13). Ranks from the CCLRS were determined by half lines according to each optimum threshold from Cox and logistic regression models using the MaxStat package (14). Nomograms were formulated based on the clinical covariates by using $\mathrm{R}$ version 3.4.4 (http://www.r-project. org/), which were assessed by calibration plots comparing actual proportion of recurrence-free survival and predicted risk probability. Time-to-recurrence was equated by fitting the CCLRS-adjusted every-three-months probabilities into one formula dependent to ranks. Comparisons between the CCLRS and preexisting staging systems were evaluated by Kaplan-Meier curves and BS. The false discovery rate for the involved covariates was controlled at $<0.05$ by the Benjamini-Hochberg procedure (15).

\section{Results}

\section{Patient characteristics and recurrence rates}

ICC patients were enrolled as described in Figure 1. The training set involved 1,323 patients with a median age of 57 years (IQR, 49 to 64 ) and $35 \%$ of female distribution (Table 1). Alcohol use, smoking, hypertension, diabetes, liver fluke, and cholelithiasis were present in $228(17 \%)$, 310 (23\%), 230 (17\%), 125 (9\%), 47 (4\%), and 115 (9\%) patients, respectively. Serologic examinations demonstrated that insufficient albumin was detected in 231 cases (17\%) and viral hepatitis infection was found in 595 (45\%) cases. Tumor size was generally large (median, $6.0 \mathrm{~cm}$ ) with preponderance of single nodule $(\mathrm{n}=1,147 ; 87 \%)$. Characteristics of the validation cohorts are shown in the Table S1. Recurrence occurred in 706 patients (53.4\%) within 1 year, 185 patients (14.0\%) between 1 to 3 years, and 15 patients $(1.1 \%)$ between 3 to 5 years in the training dataset and 161 patients (48.5\%) within 1 year, 70 patients (21.1\%) between 1 to 3 years, and 4 patients $(1.2 \%)$ between 3 to 5 years after surgical resection.

\section{Identification of prognostic covariates}

Differential coefficients of 12 covariates were associated with outcome in the univariate analysis of the training 


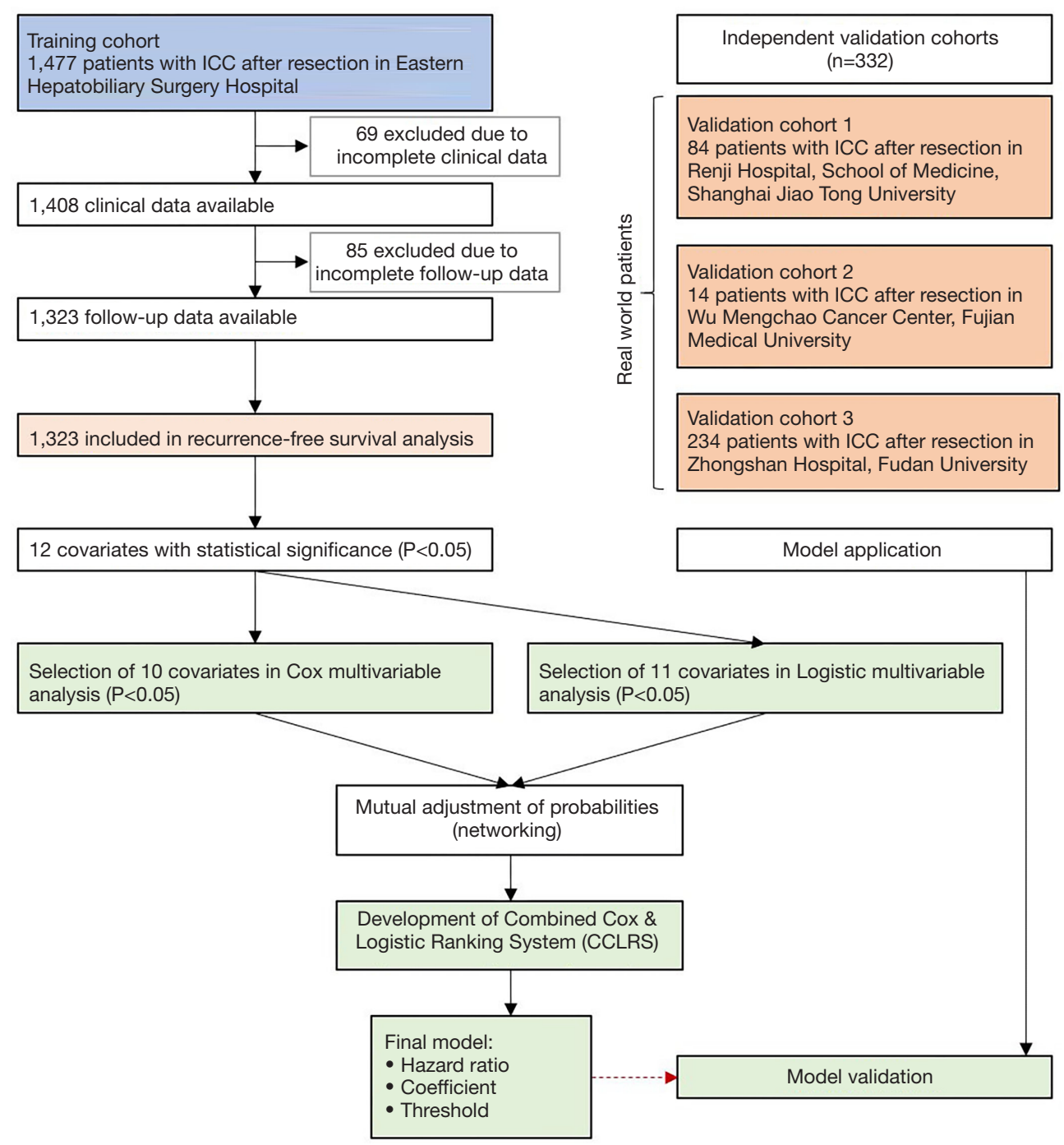

Figure 1 Outline of the study. A total of 1,323 patients with high quality of data were enrolled from 1,477 cases. Multivariable Cox and Logistic analyses identified 10 and 11 significant and independent prognostic covariates, respectively. Probability for Cox and logistic models was networked and adjusted into one probability to build a predictive signature, which was validated in three independent clinical cohorts.

cohort in both Cox proportional hazards and logistic regression models (Table S2). Among them, 10 factors showed significant in multivariable analysis, including diabetes, cholelithiasis, albumin, platelet, hepatitis B virus (HBV), AFP, CA19-9, CEA, tumor size and number, and lymph node metastasis, whereas cholelithiasis and resection type were further confirmed in the Cox multivariable analysis.

All specified covariates were employed to build a predictive signature, which were presented as nomograms (Figure S1). The model performance was higher in the logistic model [area under curve (AUC), 0.9191] compared to the Cox model (AUC, 0.9033). The hazard ratio for the Cox multivariable regression ranged from 1.16 to 2.79 for unfavorable prognostic factors, whereas the coefficients varied from 0.28 to 2.58 . The most predictive factor for recurrence was tumor size $(>5 \mathrm{~cm})$ followed by CA19-9 $(>37 \mathrm{kU} / \mathrm{L})$, in a time-dependent manner.

\section{Comparison between Cox and logistic regression models and development of the CCLRS}

The BS (0.151) was higher in Cox multivariable model (AUC, 0.9041; BS, 0.151) compared to logistic multivariable 
Table 1 Demographic and clinical characteristics of the training cohort

\begin{tabular}{|c|c|}
\hline Variable & Patients $(n=1,323)$ \\
\hline Age, years, median [IQR] & 57 [49-64] \\
\hline Sex (female) & $463(35 \%)$ \\
\hline Active alcohol use & $228(17 \%)$ \\
\hline Smoking & $310(23 \%)$ \\
\hline Hypertension & $230(17 \%)$ \\
\hline Diabetes & $125(9 \%)$ \\
\hline Liver fluke & $47(4 \%)$ \\
\hline Cholelithiasis & $115(9 \%)$ \\
\hline Albumin $<35 \mathrm{~g} / \mathrm{L}$ & $231(17 \%)$ \\
\hline \multicolumn{2}{|l|}{ Platelet count, $\times 10^{9} / \mathrm{L}$} \\
\hline$<100$ & $183(14 \%)$ \\
\hline $100-300$ & $892(67 \%)$ \\
\hline$>300$ & $248(19 \%)$ \\
\hline \multicolumn{2}{|l|}{ Viral hepatitis infection } \\
\hline Hepatitis B virus infection & $584(44 \%)$ \\
\hline Hepatitis $C$ virus infection & $11(1 \%)$ \\
\hline Cirrhosis & $301(23 \%)$ \\
\hline Etiology of liver cirrhosis & $301(100 \%)$ \\
\hline HBV infection & $259(86 \%)$ \\
\hline HCV infection & $4(1 \%)$ \\
\hline Alcoholic & $15(5 \%)$ \\
\hline Others & $23(8 \%)$ \\
\hline AFP, ng/mL, median [IQR] & $3.1[2.1-5.5]$ \\
\hline CA19-9, kU/L, median [IQR] & $20.2[3.6-81.9]$ \\
\hline CEA, ng/mL, median [IQR] & $2.8[1.7-5.7]$ \\
\hline Tumor size, cm, median [IQR] & $6.0[4.0-8.0]$ \\
\hline$\leq .0$ & $94(7 \%)$ \\
\hline $2.0-3.0$ & $131(10 \%)$ \\
\hline $3.1-5.0$ & $377(28 \%)$ \\
\hline$>5$ & $721(54 \%)$ \\
\hline \multicolumn{2}{|l|}{ Tumor number } \\
\hline 1 & $1,147(87 \%)$ \\
\hline 2 & 75 (6\%) \\
\hline$\geq 5$ & $101(8 \%)$ \\
\hline
\end{tabular}

Table 1 (continued)
Table 1 (continued)

\begin{tabular}{lc}
\hline Variable & Patients $(\mathrm{n}=1,323)$ \\
\hline Resection type & $986(75 \%)$ \\
Minor resection & $271(20 \%)$ \\
Hemihepatectomy & $66(5 \%)$ \\
Extended hemihepatectomy & $70(5 \%)$ \\
Vascular invasion & $295(22 \%)$ \\
Lymph node metastasis & \\
\hline Data are median [IQR] or $n$ (\%). Percentages may not add up to \\
100\% due to rounding. IQR, interquartile range; HBV, hepatitis \\
B virus; HBV, hepatitis C virus; AFP, alpha fetoprotein; CA19-9, \\
carbohydrate antigen 19-9; CEA, carcinoembryonic antigen.
\end{tabular}

model (BS, 0.130). However, $\mathrm{R}^{2}$, g, gr, and Dxy indices were higher in logistic model (Table S3). The identified significant covariates in the multivariable analyses were adopted to develop 2 nomograms, including Cox and logistic nomograms (Figure $2 A, B$ ). The AUC values of ROC curves were satisfactory (Figure $2 C, D$ ). Since Cox model is intended to build a predictive model dependent to time frame, the logistic model, that overperformed the Cox model was combined to the Cox model to improve predictive accuracy, which consisted the CCLRS.

In order to proceed combination of Cox \& logistic models, the two probabilities were adjusted into one probability according to the optimum thresholds from the two models for each patient in the training cohort. Moreover, the CCLRS was evaluated using Kaplan-Meier estimation according to the adjusted rank of patients and revealed to be significantly discriminative $(\mathrm{P}<0.0001$; Figure S2). For simplified application of the model and clear understanding, specific frequent circumstances with ranks and risks are provided in Table S4.

\section{Validation of the CCLRS model}

Validation of the proposed model was performed in three independent cohorts from different large clinical centers. Comparison of actual proportion of recurrence-free survival and predicted risk rank of recurrence revealed initial excellent accuracy ever overperforming all other previous predictive models for ICC (Figure 3) (validation cohort 1, Figure $3 A$; validation cohort 3, Figure $3 C$; pooled validation cohort, Figure $3 E$ ). In evaluation of discriminative capacity, 
A

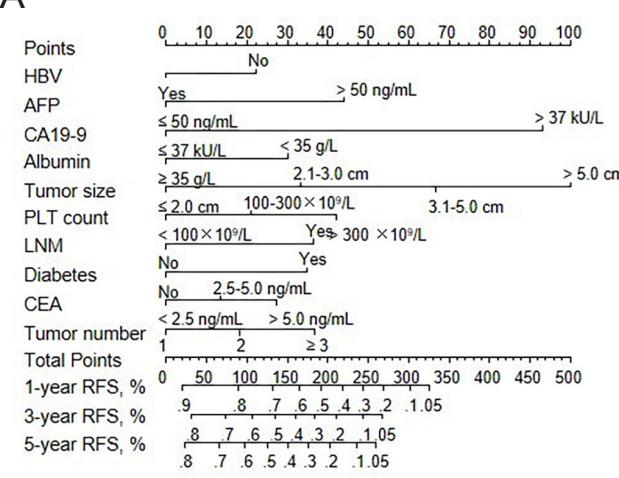

C

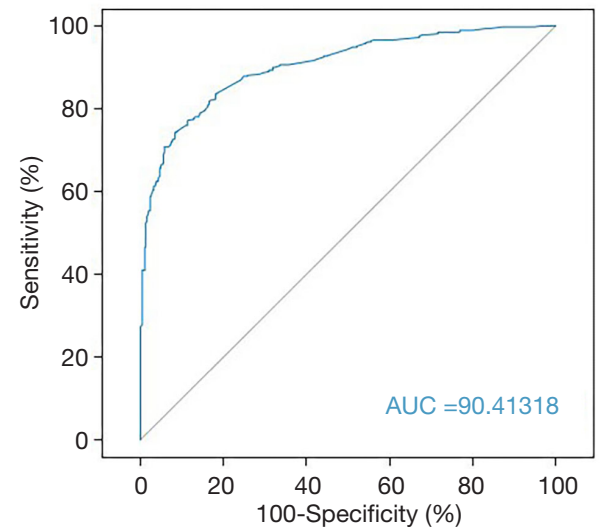

B

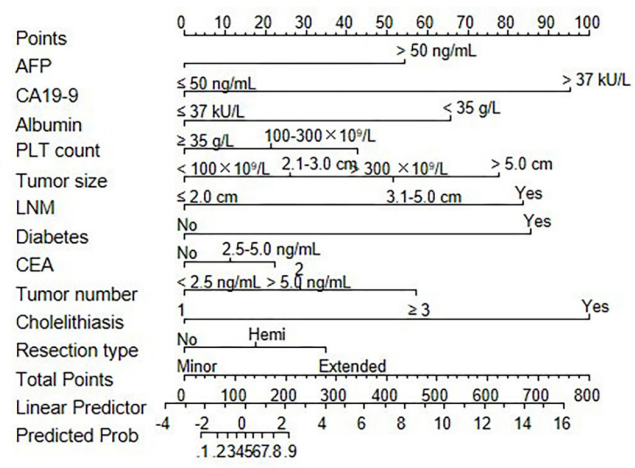

D

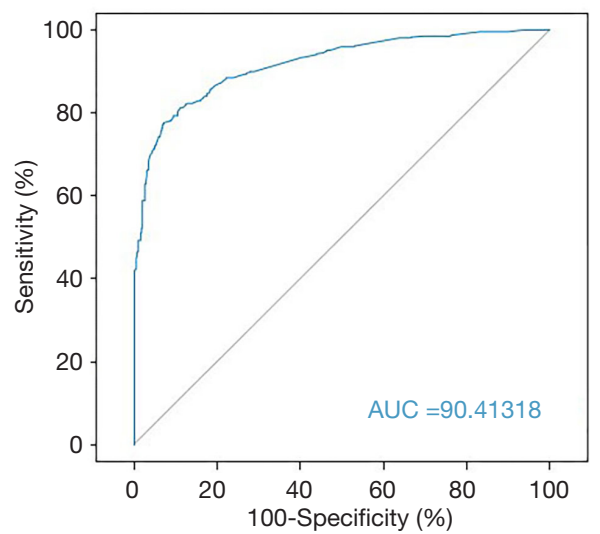

Figure 2 Nomograms generated for prediction of recurrence in patients with ICC after resection from multivariate analyses and development of the CCLRS. All significant covariates from the multivariate analyses were located on the left row and a straight line is drawn up to the points (located in the first row in each nomogram) to determine the corresponding points. Total points were added up and a straight line is drawn down to RFS rates for Cox and predicted probability for logistic models (located at the last row in each nomogram) to determine the individualized predicted survival probability. In ROC curves, thin gray lines represent the reference line. (A) A nomogram to predict recurrence of ICC using Cox regression model. (B) A nomogram to predict recurrence of ICC using logistic regression model. (C) ROC curve for the Cox regression model. (D) ROC curve for the Logistic regression model. ICC, intrahepatic cholangiocarcinoma; CCLRS, combined Cox \& logistic ranking system; RFS, recurrence-free survival; ROC, receiver operating characteristic.

incredible accuracy (AUC $>0.8$ in all validation cohorts) was found overperforming previous predictive models (AUC $<0.7)$. Specific AUC for validation cohort 1 was 0.8372 (Figure 3B), 0.8086 for validation cohort 3 (Figure 3D), and 0.8156 for pooled validation cohort (Figure $3 F$ ). Adjusted probabilities for different ranks of the CCLRS were trained as shown in Figure 4A. For demonstration of the model performance, we segmented into 7 ranks instead of 3 risk groups. Time-dependent probabilities for ranks were consistently well-distributed and rankdependent distribution was gradually narrowed according to the months after surgery. Our intent of classification was achieved excellently in the validation cohort that predicted probabilities using the CCLRS showed homogeny to the training cohort (Figure 4B). Each patient-to-actual recurrence according to the estimated CCLRS probability revealed that predominance of recurrence (marked in red) was found in patients with high probability in training (Figure $4 C$ ) and pooled validation cohort (Figure $4 D$ ). Specific median survival, 1 - and 5-year recurrence-free survival with adjusted hazard ratios for each rank are shown in Table 2. In addition, rank-dependent clinical characteristics of patients in the validation cohorts were assessed to verify influential factors for the ranking system (Table S5). To support simplified use in clinical practice with intent to discriminate, all patients were stratified into low (rank 
A

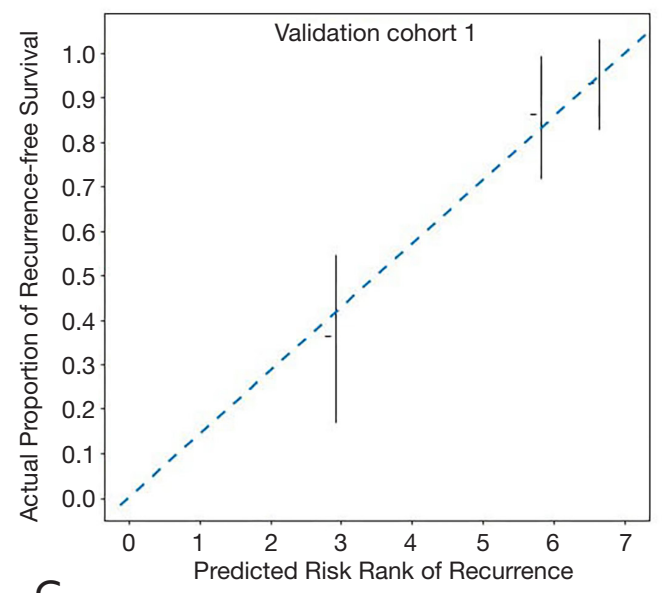

C

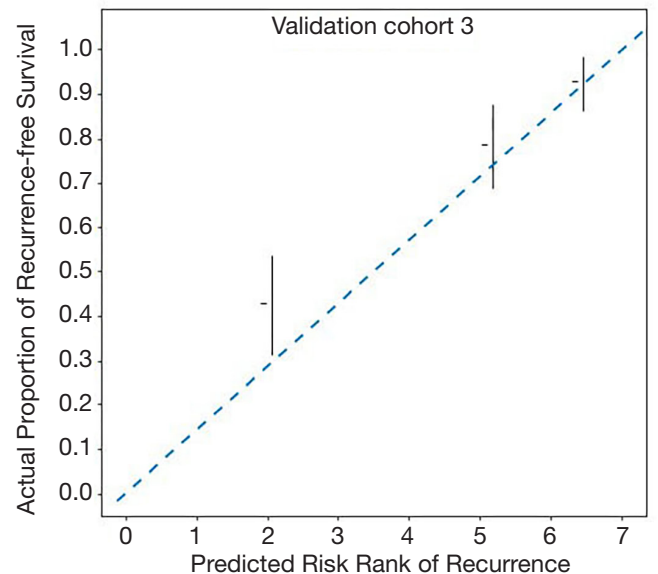

$\mathrm{E}$

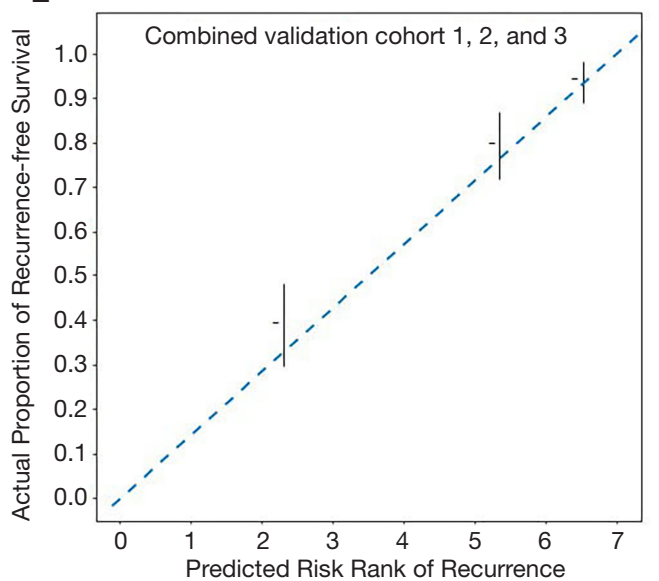

B

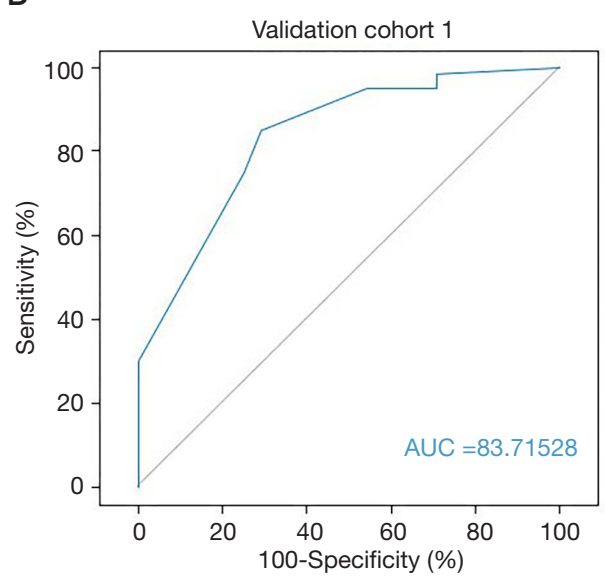

D

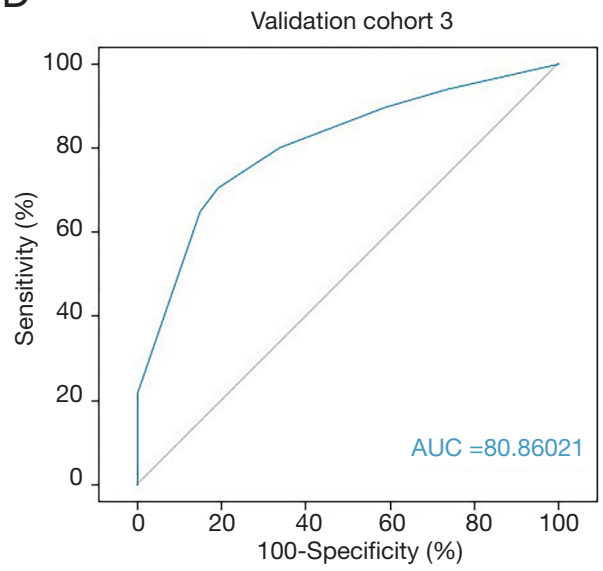

$\mathrm{F}$

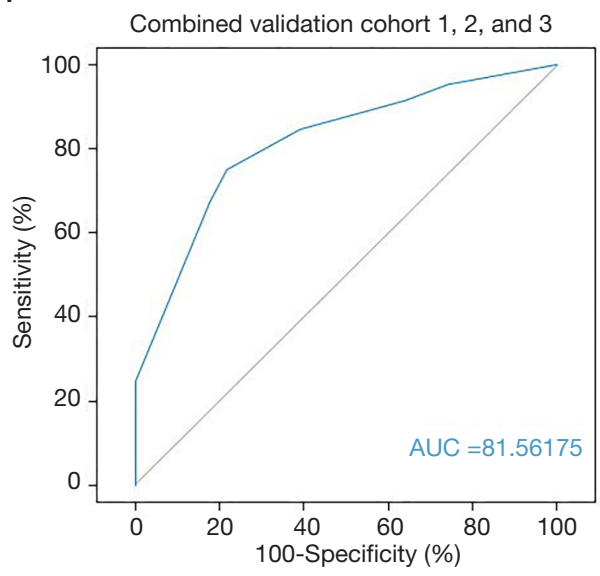

Figure 3 Validation of the CCLRS. The results obtained from the training set were validated in two independent validation cohorts drawn from Renji Hospital (validation cohort 1) and Zhongshan Hospital (validation cohort 3). Independent evaluation of the validation cohort 2 was discarded due to small sample size; this cohort was included in the combined validation cohorts. Blue dotted and gray thin lines represent the reference lines in calibration plots and ROC curves. Shown are calibration plots comparing predicted and actual probability of recurrence-free survival in validation cohort 1 (A), validation cohort $3(\mathrm{C})$, and combined validation cohort (E), and ROC curves for evaluation accuracy in validation cohort 1 (B), validation cohort 3 (D), and combined validation cohort (F). CCLRS, combined Cox \& logistic ranking system; ROC, receiver operating characteristic. 

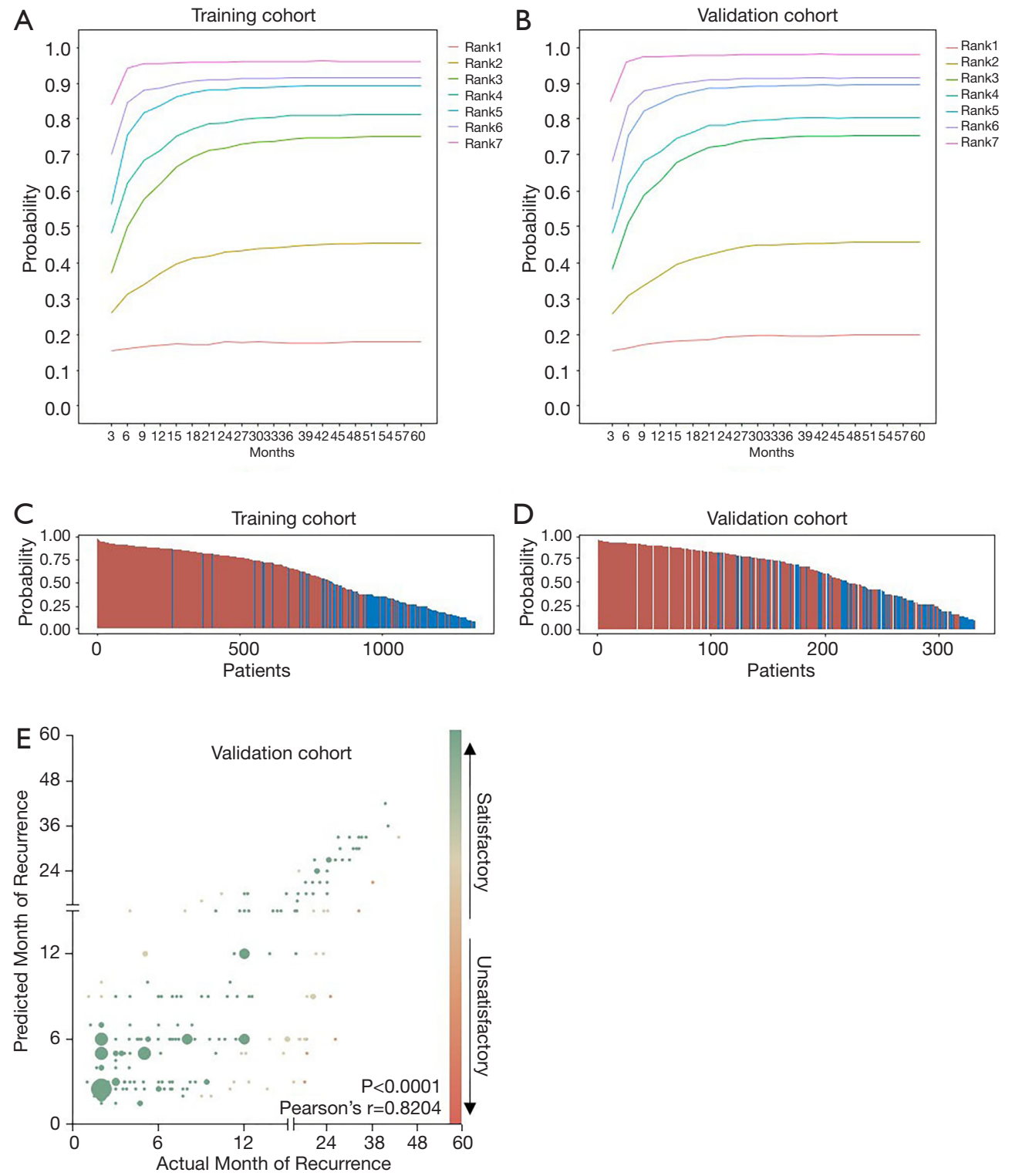

Figure 4 Evaluation of model performance for rank-dependent predicted probabilities, probability-dependent actual outcomes, and estimated time-to-recurrence. Rank-dependent adjusted probabilities obtained from the CCLRS were assessed using Kaplan-Meier estimation for discriminative ability in both training cohort (A) and validation cohort (B). Patient-to-recurrence columns (patients with recurrence, marked in red; patients without recurrence, marked in blue) in training cohort (C) and validation cohort (D) were generated for validation of model fit. Estimation of time-to-recurrence was tested in the validation cohort and obtained Pearson $\mathrm{r}$ value of 0.8204 ( $\mathrm{P}<0.0001$; E). Size of each plot is in proportion to the number of overlapping patients. CCLRS, combined Cox \& logistic ranking system.

1-2), moderate (rank 3-5), and high (rank 6-7) risk groups according to the risk probability for recurrence (Table S6). The median survival for the low, moderate, and high groups revealed to be $3.2,1.3$, and 0.6 years, respectively, with significant between-group differences $(\mathrm{P}<0.001)$.

\section{Calculation of time-to-recurrence with CCLRS model}

In order to evaluate its capacity in prediction of specific time to recurrence after resection, we developed a CCLRSbased formula by fitting the CCLRS-adjusted every-three- 


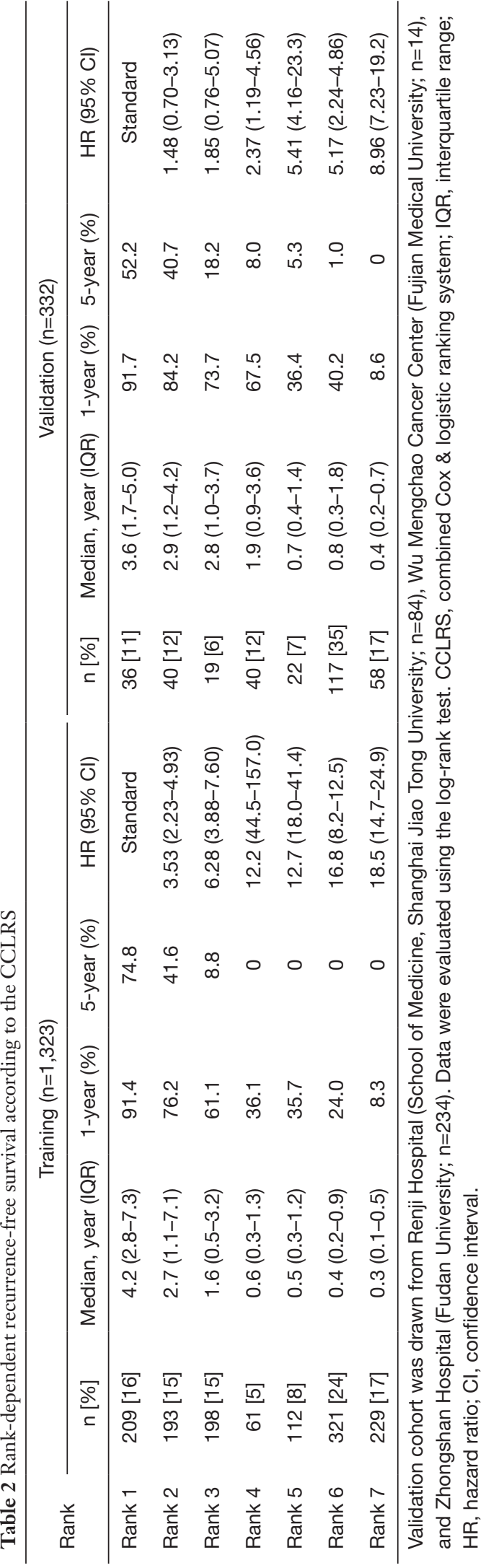

months probabilities of 1323 individuals from the training dataset into one formula dependent to ranks: If rank 3 in $f^{-1}$ $(0.5)$; if $2<\operatorname{rank}<7,\left[f^{-1}(0.01)\right]^{-1}$; if rank=7, 13.5-19 $f(\mathrm{x})$; where the unit is month The developed rank-dependent formula was tested in the combined validation cohorts and achieved a correlation index (r) of 0.8204 with an error of \pm 0.5 years $(\mathrm{P}<0.0001$; Figure $4 E)$.

\section{Comparison of The CCLRS with preexisting staging systems}

The CCLRS probability for patients with ICC after resection was compared with preexisting ICC staging systems, including American Joint Committee on Cancer (AJCC) eighth edition, AJCC seventh edition, Hyder nomogram (8), Liver Cancer Study Group of Japan (LCSGJ) (16), Japanese Society of Hepato-Biliary Pancreatic Surgery (JSHBPS) (17), and the CCLRS for evaluation of discriminative capacity (Figure S3). For Hyder nomogram and the CCLRS, the calculated probabilities were classified into 4 stages for simulation of same condition. Superior stage-dependent recurrence-free survival was observed in the CCLRS compared to other systems $(\mathrm{P}<0.0001)$. In addition, calibration plots were generated to evaluate consistency between actual and predicted proportion of recurrence-free survival (Figure S4). The BS was also superior in the CCLRS (0.107), overperforming AJCC eighth edition (0.320), AJCC seventh edition (0.299), Hyder nomogram (0.400), LCSGJ (0.136), and JSHBPS (0.153).

\section{Discussion}

We have developed a predictor for recurrence of ICC after resection based on clinical factors. Accuracy and discriminative abilities were significantly improved by developing a network between Cox and logistic regression models, mutually adjusting risk probabilities, which was validated by three clinical cohorts.

The Cox proportional hazards regression model has been extensively applied in prediction of survival outcome using the superiority in achieving time-dependent risk, whereas the logistic regression is frequently used in the development of diagnostic or dual-outcome models using the superiority of predictive accuracy $(18,19)$. Our attempt to combine Cox and logistic models was made after performing multivariable analyses using the two methodologies. The two methodologies demonstrated different independent significant factors and their prognostic impact also differed. These differences enlightened us to consider that predictive 
performance may improve if combine the two regression models using mutual networking. Unexpectedly, networking of the two models significantly improved sensitivity and specificity, confirmed by a minimum AUC of 0.8086 among three clinical validation cohorts.

Among the included covariates, tumor size and number, lymph node metastasis, type of resection, CA19-9, CEA (20), diabetes (21), cholelithiasis (22), albumin (23), and HBV infection (24) are previously reported prognostic factors. However, prognostic impact of serum platelet count remains further confirmation. Recently, platelet-derived growth factor $\mathrm{D}$ was found to be associated with the recruitment and expansion of cancer-associated fibroblast, contributing to invasion and metastasis in ICC (25). Furthermore, CD24, a ligand for an adhesion receptor on platelets that enhances the metastatic potential of cancers, was found to be an independent prognostic factor for ICC (26). Moreover, AFP, which is a representative diagnostic and prognostic marker for hepatocellular carcinoma, and a distinctive feature of $\mathrm{HBV}$-associated ICC, was identified as an independent predictor that AFP elevation to a prognostic level $(>50 \mathrm{ng} / \mathrm{mL})$ showed significant association with recurrence of the tumor in patients with ICC after resection. Elevation of AFP in $\mathrm{HBV}$-associated ICC as a significant factor for recurrence of the tumor seems to be potentially due to the fact that HBV-associated ICC and hepatocellular carcinoma hold common disease process (27). Alike hepatocellular carcinomas, that AFP elevation is a common prognostic factor for recurrence of the tumor after resection, AFP elevation also predicted recurrence in $\mathrm{HBV}$-associated ICC after resection in the present study. A recent study by Yang et al. (28) indicated that HBV-caused inflammation represented by HBV-related parameters, including AFP, play a major role in inducing ICC. This impact was confirmed in a recent study of $\mathrm{HBV}$-associated ICC that serum AFP level was identified as an independent unfavorable prognostic factor after resection (29). However, our study is first to identify and implement AFP elevation as a significant prognostic factor with study population of both $\mathrm{HBV}$-associated and non-HBV-associated ICC, but the prognostic nomogram may be limited to be used in areas with high proportion of HBV-associated ICC, which awaits future validation by other regions with different etiologic proportion of ICCs.

In this study, the predictors of recurrence-free survival in patients with ICC actively excluded other staging systems, such as AJCC and LCSGJ stages, to facilitate our model with intend to develop a single tool that would be easily applied in real-world clinical setting. Thus, the covariates for our model included diabetes, cholelithiasis, serum albumin and platelet, HBV, AFP, CA19-9, CEA, tumor size and number, lymph node metastasis, and type of resection. All these factors were categorized into 2 to 4 categories quantitatively in accordance to general cut-off for elevation (30-32).

We also compared the CCLRS with preexisting staging systems, including AJCC eighth and seventh editions, Hyder nomogram, LCSGJ, and JSHBPS to investigate the competitivity. Statistically, the CCLRS was superior in terms of accuracy and discrimination overperforming all other staging systems. Recently, Bagante et al. (33) also suggested that the preexisting nomograms based on clinicopathologic characteristics are suboptimal in accurate prediction of recurrence by validating models in 897 patients with ICC after curative-intent resection, which may be totally or partially due to limited methodologic approaches that allow precise predictive evaluation.

Another important concern is genetic mutations, such as Kras and TP53, that were found to cause cholangiocarcinoma (34). Some mutations, including TP53 $(\mathrm{P}=0.0031)$ and ARID1A $(\mathrm{P}=0.0007)$, were previously identified as independent predictors of poor prognosis in patients with ICC after resection (35). In a precise prognostic prediction setting, inclusion of genetic mutation may significantly improve the model performance, which was not involved in the present study due to data availability. Therefore, we call for both comparison of predictive ability between our model and genetic mutations, and combination of genetic mutations and our model since it seems to have potential to improve predictive ability.

Despite novelties in accuracy and initial attempt to predict time-to-recurrence after resection, our model has several underlying limitations. Although ICC is a tumor of early recurrence even after surgical resection, not all patients in both training and validation cohorts completed 5 years of follow-up, thus application of logistic regression is not conventional. The CCLRS is a network system that assessed risk probabilities of risk from the two regressions are interactive and mutually adjusted, thus we hypothesized that this multilayered estimation allows inclusion of partial censored patients. The proportion of patients with multiple tumors needs to be considered when interpreting our results. In the validation cohort 1 and 3,17\% patients had $\geq 3$ tumors. In addition, the etiologic subtypes of the study cohort need to be considered when interpreting our results. The proportions of smoking, diabetes, HBV infection, and liver cirrhosis were $23 \%, 9 \%, 44 \%$, and $23 \%$, but only $1 \%$ of the patients had HCV infection. Therefore, validation of 
the results is required for the model to be applied in regions with different proportions of etiologic subtypes, especially in Western populations. In addition, there were patients with lymph node metastasis in both training and validation cohorts. Future validation of the model by cohorts without lymph node metastasis would be required for our model to be applied in cohorts without regional practice. Lastly, application of the model may seem complex to be used in clinical medicine, especially for those who are not fluent in calculating nomogram scores. However, it would become simple after trying a few samples. From our point of view, inclusion of less factors provides easy use with limited accuracy, thus relatively complicated approach is essential in achieving accurate predictive performance.

In conclusion, we have established the CCLRS to stratify patients with ICC after resection into 7 ranks with distinct time-dependent risk probabilities. This model captures multiple aspects of the prognostic factors of ICC and the heterogenous composition of the prognostic coefficient. For patients with high ranks, intensive follow-up may be warranted. In addition, surgical treatment may not provide survival benefits in this group of patients. Furthermore, our attempt to predict specific time-to-recurrence was realized for the first time in literature and resulted in satisfactory accuracy. In addition, methodology of the CCLRS may also improve performance of predictive tools in other diseases and/or treatment modalities. Collectively, the CCLRS for ICC is a promising tool with superiority to predict individualized risk of recurrence and to estimate timedependent disease status after resection.

\section{Acknowledgments}

Funding: This study was supported by the National Key R\&D Program of China (2017YFA0505803, 2018ZX10732202-001-001), National Key Research on Precision Medicine of China (2017YFC0908102), and Top Priority Clinical Medical Center of Shanghai Health and Family Planning Commission (2017ZZ01018).

\section{Footnote}

Reporting Checklist: The authors have completed the TRIPOD reporting checklist. Available at https://hbsn. amegroups.com/article/view/10.21037/hbsn.2020.01.07/rc

Data Sharing Statement: Available at https://hbsn. amegroups.com/article/view/10.21037/hbsn.2020.01.07/dss
Conflicts of Interest: All authors have completed the ICMJE uniform disclosure form (available at https://hbsn. amegroups.com/article/view/10.21037/hbsn.2020.01.07/coif). The authors have no conflicts of interest to declare.

Etbical Statement: The authors are accountable for all aspects of the work in ensuring that questions related to the accuracy or integrity of any part of the work are appropriately investigated and resolved. The study was conducted in accordance with the Declaration of Helsinki (as revised in 2013). This study was a retrospective analysis of anonymous clinical data and, according to institutional guidelines, did not require approval by the institutional research ethics board. All patients gave informed consent to surgery as well as the use of their anonymous clinical data for research purposes.

Open Access Statement: This is an Open Access article distributed in accordance with the Creative Commons Attribution-NonCommercial-NoDerivs 4.0 International License (CC BY-NC-ND 4.0), which permits the noncommercial replication and distribution of the article with the strict proviso that no changes or edits are made and the original work is properly cited (including links to both the formal publication through the relevant DOI and the license). See: https://creativecommons.org/licenses/by-nc-nd/4.0/.

\section{References}

1. Razumilava N, Gores GJ. Cholangiocarcinoma. Lancet 2014;383:2168-79.

2. Shaib Y, El-Serag HB. The epidemiology of cholangiocarcinoma. Semin Liver Dis 2004;24:115-25.

3. Saha SK, Zhu AX, Fuchs CS, et al. Forty-Year Trends in Cholangiocarcinoma Incidence in the U.S.: Intrahepatic Disease on the Rise. Oncologist 2016;21:594-9.

4. Mavros MN, Economopoulos KP, Alexiou VG, et al. Treatment and Prognosis for Patients With Intrahepatic Cholangiocarcinoma: Systemic Review and Meta-analysis. JAMA Surg 2014;149:565-74.

5. Cox DR. The regression analysis of binary sequences (with discussion). J Roy Stat Soc B 1958;20:215-22.

6. Cox DR. Regression Models and Life-Tables. J Roy Stat Soc B 1972;34:187-220.

7. Hyder $\mathrm{O}$, Marques $\mathrm{H}$, Pulitano $\mathrm{C}$, et al. A nomogram to predict long-term survival after resection for intrahepatic cholangiocarcinoma: an Eastern and Western experience. JAMA Surg 2014;149:432-8.

8. Wang Y, Li J, Xia Y, et al. Prognostic nomogram for intrahepatic cholangiocarcinoma after partial hepatectomy. 
J Clin Oncol 2013;31:1188-95.

9. Ali SM, Clark CJ, Mounajjed T, et al. Model to predict survival after surgical resection of intrahepatic cholangiocarcinoma: the Mayo Clinic experience. HPB (Oxford) 2015;17:244-50.

10. Buettner S, Galjart B, van Vugt JLA, et al. Performance of prognostic scores and staging systems in predicting long-term survival outcomes after surgery for intrahepatic cholangiocarcinoma. J Surg Oncol 2017;116:1085-95.

11. Collins GS, Reitsma JB, Altman DG, et al. Transparent reporting of a multivariable prediction model for individual prognosis or diagnosis (TRIPOD): the TRIPOD statement. BMJ 2015;350:g7594.

12. Sempoux C, Jibara G, Ward SC, et al. Intrahepatic cholangiocarcinoma: New insights in pathology. Semin Liver Dis 2011;31:49-60.

13. Uno H, Cai T, Pencina MJ, et al. On the C-statistics for evaluating overall adequacy of risk prediction procedures with censored survival data. Stat Med 2011;30:1105-17.

14. Hothorn T, Lausen B. On maximally selected rank statistics. R News 2002;2:3-5.

15. Benjiamini $Y$, Hochberg Y. Controlling the false discovery rate: a practical and powerful approach to multiple testing. Journal of the Royal Statistical Society, Series B 1995;57:289-300.

16. Yamasaki S. Intrahepatic cholangiocarcinoma: macroscopic type and stage classification. J Hepatobiliary Pancreat Surg 2003;10:288-91.

17. Uenishi T, Ariizumi S, Aoki T, et al. Proposal of a new staging system for mass-forming intrahepatic cholangiocarcinoma: a multicenter analysis by the Study Group for Hepatic Surgery of the Japanese Society of Hepato-Biliary-Pancreatic Surgery. J Hepatobiliary Pancreat Sci 2014;21:499-508.

18. Fisher LD, Lin DY. Time-dependent covariates in the Cox proportional-hazards regression model. Annu Rev Public Health 1999;20:145-57.

19. Stoltzfus JC. Logistic regression: a brief primer. Acad Emerg Med 2011;18:1099-104.

20. He C, Zhang Y, Song Y, et al. Preoperative CEA levels are supplementary to CA19-9 levels in predicting prognosis in patients with resectable intrahepatic cholangiocarcinoma. J Cancer 2018;9:3117-28.

21. Liu RQ, Shen SJ, Hu XF, et al. Prognosis of the intrahepatic cholangiocarcinoma after resection: hepatitis $B$ virus infection and adjuvant chemotherapy are favorable prognosis factors. Cancer Cell Int 2013;13:99.

22. Kim HJ, Kim JS, Joo MK, et al. Hepatolithiasis and intrahepatic cholangiocarcinoma: A review. World J Gastroenterol 2015;21:13418-31.

23. Zhang C, Wang H, Ning Z, et al. Serum liver enzymes serve as prognostic factors in patients with intrahepatic cholangiocarcinoma. Onco Targets Ther 2017;10:1441-9.

24. Jeong S, Luo G, Wang ZH, et al. Impact of viral hepatitis
B status on outcomes of intrahepatic cholangiocarcinoma: a meta-analysis. Hepatol Int 2018;12:330-8.

25. Sirica AE, Gores GJ, Groopman JD, et al. Intrahepatic Cholangiocarcinoma: Continuing Challenges and Translational Advances. Hepatology 2019;69:1803-15.

26. Su MC, Hsu C, Kao HL, et al. CD24 expression is a prognostic factor in intrahepatic cholangiocarcinoma. Cancer Lett 2006;235:34-9.

27. Zhou H, Wang H, Zhou D, et al. Hepatitis B virusassociated intrahepatic cholangiocarcinoma and hepatocellular carcinoma may hold common disease process for carcinogenesis. Eur J Cancer 2010;46:1056-61.

28. Yang F, Ma L, Yang Y, et al. Contribution of Hepatitis B Virus Infection to the Aggressiveness of Primary Liver Cancer: A Clinical Epidemiological Study in Eastern China. Front Oncol 2019;9:370.

29. Huang J, Wang X, Zhu Y, et al. Specific prognostic factors in hepatitis B virus-related and non-hepatitis B virusrelated intrahepatic cholangiocarcinoma after macroscopic curative resection. J Surg Oncol 2019;119:40-6.

30. Yamamoto Y, Hosogaya S, Osawa S, et al. Nationwide multicenter study aimed at the establishment of common reference intervals for standardized clinical laboratory tests in Japan. Clin Chem Lab Med 2013;51:1663-72.

31. Sizaret P, Martel N, Tuyns A, et al. Mean alphafetoprotein values of 1,333 males over 15 years by age groups. Digestion 1977;15:97-103.

32. Ballesta AM, Molina R, Filella X, et al. Carcinoembryonic antigen in staging and follow-up of patients with solid tumors. Tumour Biol 1995;16:32-41.

33. Bagante F, Merath K, Squires MH, et al. The Limitations of Standard Clinicopathologic Features to Accurately Risk-Stratify Prognosis after Resection of Intrahepatic Cholangiocarcinoma. J Gastrointest Surg 2018;22:477-85.

34. Hill MA, Alexander WB, Guo B, et al. Kras and Tp53 Mutations Cause Cholangiocyte- and Hepatocyte-Derived Cholangiocarcinoma. Cancer Res 2018;78:4445-51.

35. Simbolo M, Vicentini C, Ruzzenente A, et al. Genetic alterations analysis in prognostic stratified groups identified TP53 and ARID1A as poor clinical performance markers in intrahepatic cholangiocarcinoma. Sci Rep 2018;8:7119.

Cite this article as: Jeong S, Luo G, Gao Q, Chen J, Liu X, Dong L, Zhang Y, Shen F, Cheng Q, Sui C, Liu J, Wang H, Xia Q, Chen L. A combined Cox and logistic model provides accurate predictive performance in estimation of timedependent probabilities for recurrence of intrahepatic cholangiocarcinoma after resection. HepatoBiliary Surg Nutr 2021;10(4):464-475. doi: 10.21037/hbsn.2020.01.07 


\section{Supplementary}

Table S1 Baseline characteristics of the patients in the validation cohorts

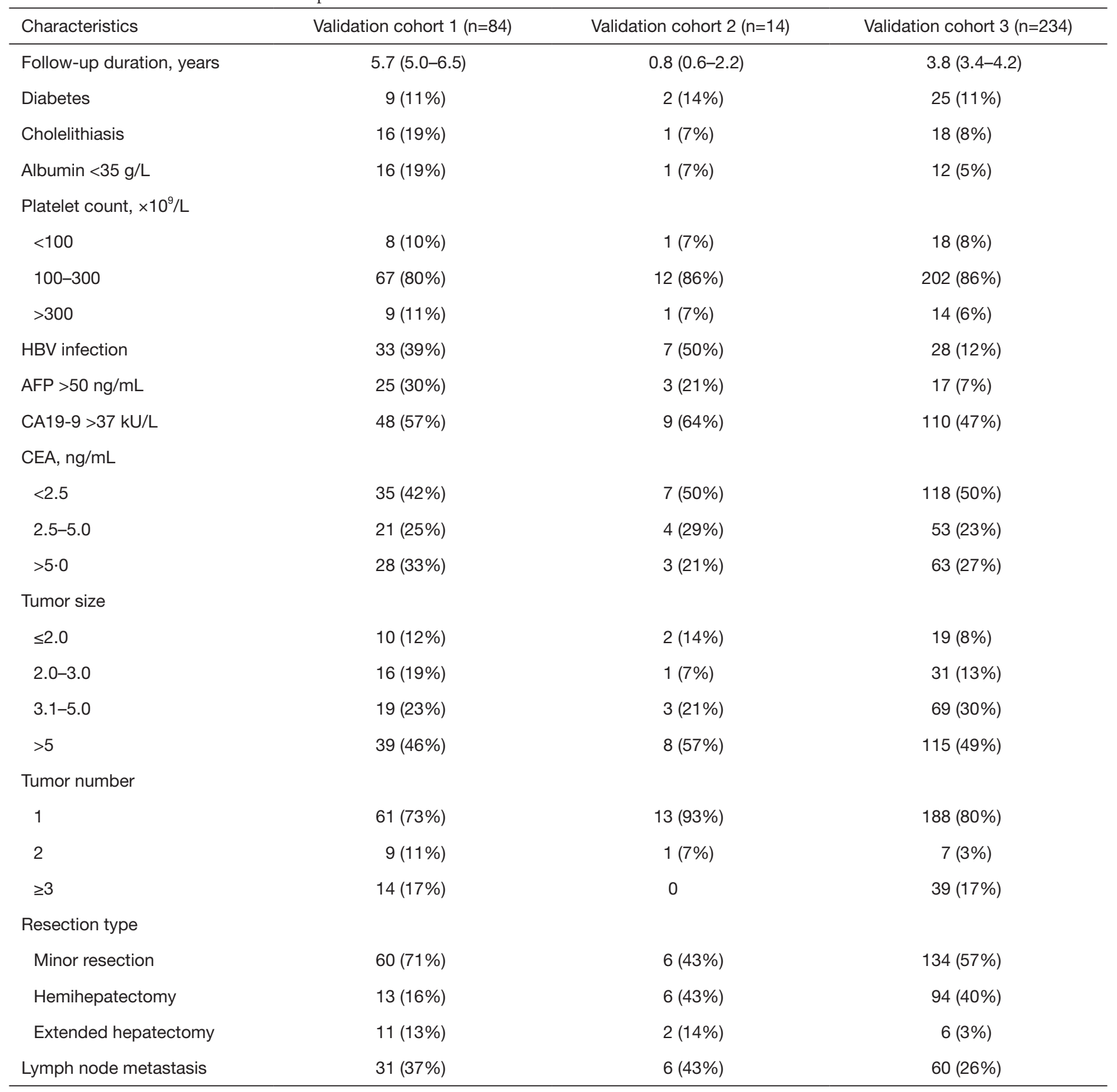

Data are median (IQR) or $\mathrm{n}(\%)$. Percentages may not add up to $100 \%$ due to rounding. The results obtained from the training cohort were validated in three independent cohorts drawn from School of Medicine, Shanghai Jiao Tong University (Renji Hospital; validation cohort 1), Fujian Medical University (Mengchao Hepatobiliary Hospital; validation cohort 2), and Fudan University (Zhongshan Hospital; validation cohort 3). IQR, interquartile range; HBV, hepatitis B virus; AFP, alpha fetoprotein; CA19-9, carbohydrate antigen 19-9; CEA, carcinoembryonic antigen. 
Table S2 Multivariate analyses of Cox and logistic models

\begin{tabular}{lcccccc}
\hline Variable & $\mathrm{SE}^{\mathrm{a}}$ & $\mathrm{HR}(95 \% \mathrm{Cl})^{\mathrm{a}}$ & $\mathrm{P}^{\mathrm{a}}$ & $\mathrm{SE}^{\mathrm{b}}$ & $\mathrm{Coef}^{\mathrm{b}}$ & $\mathrm{P}^{\mathrm{b}}$ \\
\hline Diabetes & 0.10 & $1.47(1.22-1.78)$ & $<0.001$ & 0.37 & 2.12 & $<0.001$ \\
Cholelithiasis & 0.11 & $1.01(0.82-1.25)$ & 0.914 & 0.67 & 2.58 & $<0.001$ \\
Albumin $<35 \mathrm{~g} / \mathrm{L}$ & 0.09 & $1.38(1.17-1.63)$ & $<0.001$ & 0.41 & 1.70 & $<0.001$ \\
Platelet count, $\times 10^{9} / \mathrm{L}$ & 0.06 & $1.27(1.12-1.44)$ & $<0.001$ & 0.17 & 0.55 & 0.002 \\
HBV infection & 0.07 & $0.78(0.69-0.90)$ & $<0.001$ & 0.17 & -0.24 & 0.165 \\
AFP $>50 \mathrm{ng} / \mathrm{mL}$ & 0.10 & $1.61(1.32-1.97)$ & $<0.001$ & 0.39 & 1.44 & $<0.001$ \\
CA19-9 $>37 \mathrm{kU} / \mathrm{L}$ & 0.08 & $2.79(2.38-3.27)$ & $<0.001$ & 0.23 & 2.41 & $<0.001$ \\
CEA, ng/mL & 0.04 & $1.16(1.07-1.27)$ & $<0.001$ & 0.11 & 0.28 & 0.012 \\
Tumor size, cm & 0.05 & $1.44(1.31-1.59)$ & $<0.001$ & 0.10 & 0.65 & $<0.001$ \\
Tumor number & 0.06 & $1.23(1.11-1.37)$ & $<0.001$ & 0.21 & 0.73 & 0.001 \\
Resection type & 0.06 & $0.95(0.84-1.06)$ & 0.363 & 0.20 & 0.40 & 0.041 \\
Lymph node metastasis & 0.08 & $1.51(1.29-1.76)$ & $<0.001$ & 0.34 & 2.11 & $<0.001$ \\
\hline
\end{tabular}

${ }^{a}$, calculated using Cox regression; ${ }^{b}$, calculated using logistic regression. SE, standard error; HR, hazard ratio; Cl, confidence interval; HBV, hepatitis B virus; AFP, alpha fetoprotein; CA19-9, carbohydrate antigen 19-9; CEA, carcinoembryonic antigen.

A
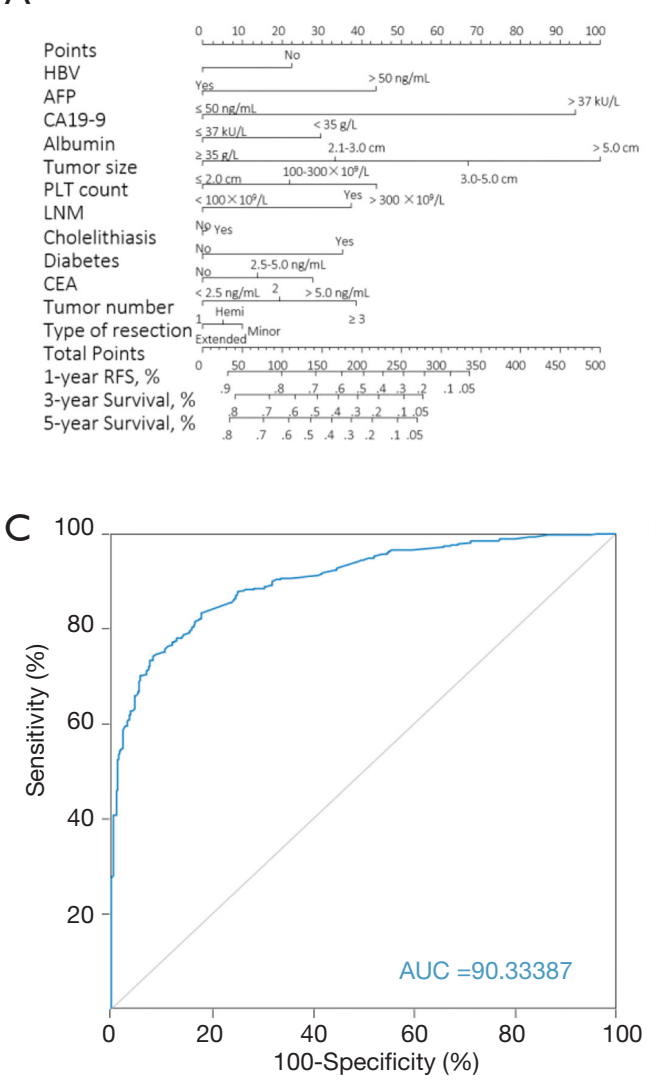

B

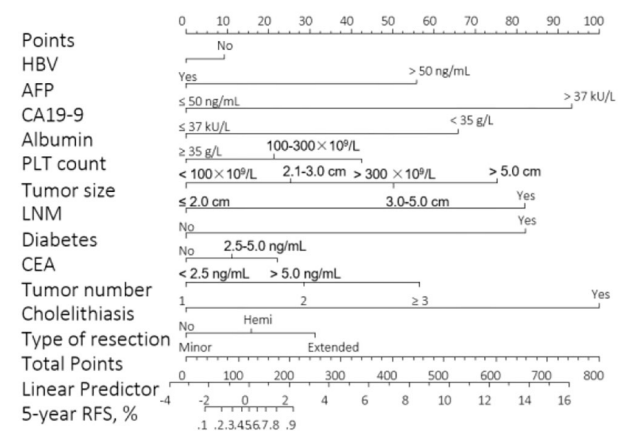

$\mathrm{D}$

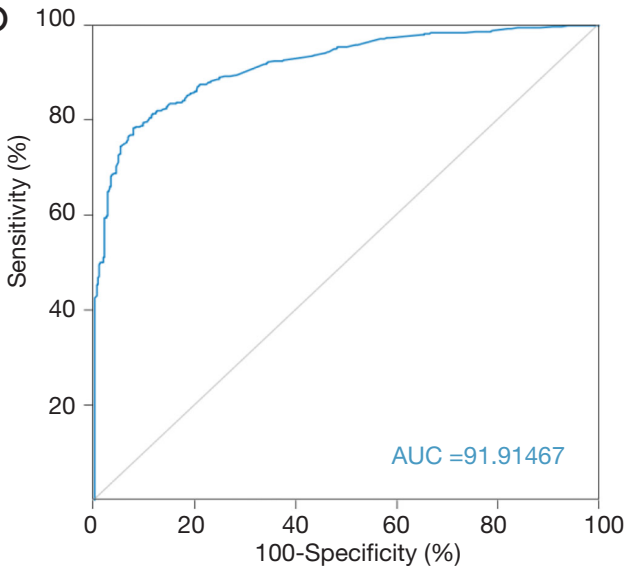

Figure S1 Development and evaluation of nomograms for prediction of recurrence of intrahepatic cholangiocarcinoma after resection using significant covariates from univariate analyses. To calculate predicted survival, all significant factors from the univariate analyses were located on the left row and a straight line is drawn up to the points to determine the corresponding points. Total points were matched to "1-year Survival, \%", "3-year Survival, \%", and "5-year Survival, \%" or "Linear Predictor" with "Predicted Value, \%" to determine the individualized predicted survival probability. (A) Cox regression model. (B) Logistic regression model. (C) ROC curve for Cox univariate regression model. (D) ROC curve for logistic univariate regression model. AUC, area under curve; ROC, receiver operating characteristic. 
Table S3 Calibration and discrimination of Cox and logistic regression models

\begin{tabular}{lcccccc}
\hline Variable & $\mathrm{R}^{2}$ & $\mathrm{~g}$ & $\mathrm{gr}$ & $\mathrm{Brier}$ & $\mathrm{C}$ & $\mathrm{Dxy}$ \\
\hline Cox univariate model & 0.428 & 1.168 & 3.215 & 0.168 & 0.9033 \\
Cox multivariate model & 0.428 & 1.164 & 3.204 & 0.151 & 0.9041 \\
Logistic univariate model & 0.622 & 3.684 & 39.814 & 0.107 & 0.9191 \\
Logistic multivariate model & 0.621 & 3.672 & 39.327 & 0.130 & 0.9186 & 0.838 \\
\hline
\end{tabular}

Gr, g-index on the odds ratio scale.

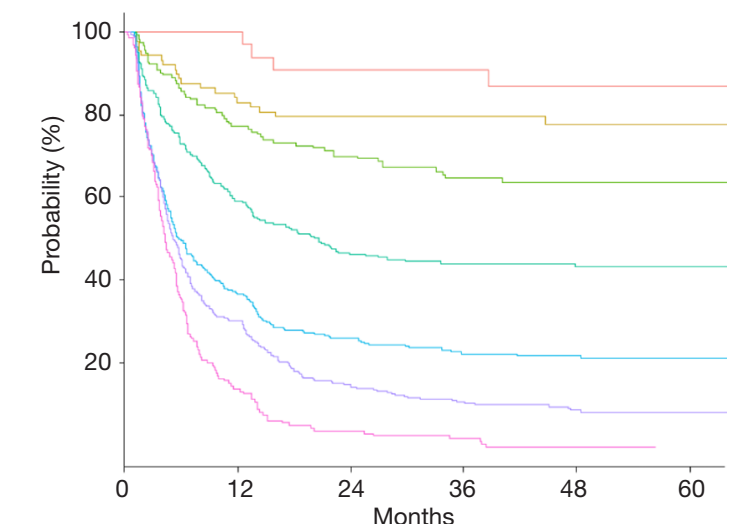

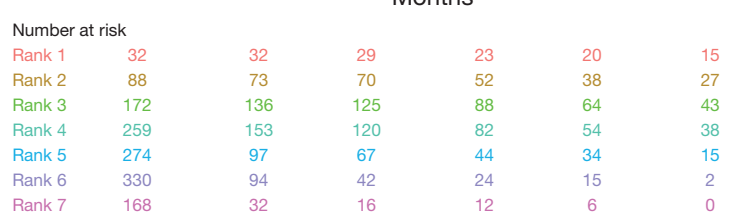

Figure S2 Kaplan-Meier estimation for recurrence-free survival of the training cohort according to ranks stratified by the CCLRS. CCLRS, combined Cox \& logistic ranking system. 
Table S4 Common circumstances and the corresponding ranks and risks according to the CCLRS

\begin{tabular}{|c|c|c|c|c|c|c|c|c|c|c|c|c|c|}
\hline $\begin{array}{l}\text { Tumor size } \\
(\mathrm{cm})\end{array}$ & $\begin{array}{c}\text { CA19-9 (kU/ } \\
\text { L) }\end{array}$ & $\begin{array}{l}\text { Albumin } \\
(\mathrm{g} / \mathrm{L})\end{array}$ & $\begin{array}{l}\text { Platelet } \\
\left(10^{9} / \mathrm{L}\right)\end{array}$ & HBV & $\begin{array}{l}\text { Lymph node } \\
\text { metastasis }\end{array}$ & Cholelithiasis & Diabetes & $\begin{array}{c}\text { CEA } \\
(\mathrm{ng} / \mathrm{mL})\end{array}$ & $\begin{array}{c}\text { AFP } \\
(\mathrm{ng} / \mathrm{mL})\end{array}$ & $\begin{array}{l}\text { Resection } \\
\text { type }\end{array}$ & $\begin{array}{l}\text { Tumor } \\
\text { number }\end{array}$ & Rank & Risk \\
\hline$\leq 2.0$ & $\leq 37$ & $\geq 35$ & Any & Any & Absent & Absent & Absent & $\leq 5.0$ & $\leq 50$ & Minor & 1 & 1 & Low \\
\hline $2.1-3.0$ & $\leq 37$ & $\geq 35$ & $\leq 300$ & Present & Absent & Absent & Absent & $\leq 5.0$ & $\leq 50$ & Minor & 2 & 1 & Low \\
\hline$\leq 2.0$ & $\leq 37$ & $\geq 35$ & $\leq 300$ & Any & Absent & Absent & Absent & $\leq 5.0$ & $>50$ & Minor & 1 & 2 & Low \\
\hline $2.1-3.0$ & $\leq 37$ & $\geq 35$ & $>300$ & Any & Absent & Absent & Absent & $<2.5$ & $\leq 50$ & Hemi & 1 & 2 & Low \\
\hline $3.1-5.0$ & $\leq 37$ & $\geq 35$ & $\leq 300$ & Any & Absent & Absent & Absent & $<2.5$ & $\leq 50$ & Minor & 1 & 2 & Low \\
\hline $2.1-3.0$ & $\leq 37$ & $\geq 35$ & $\leq 300$ & Any & Present & Absent & Absent & $>5.0$ & $\leq 50$ & Minor & 1 & 3 & Moderate \\
\hline $3.1-5.0$ & $\leq 37$ & $\geq 35$ & $\leq 300$ & Absent & Absent & Absent & Absent & $>5.0$ & $\leq 50$ & Hemi & 1 & 3 & Moderate \\
\hline $2.1-3.0$ & $\leq 37$ & $\geq 35$ & $\leq 300$ & Any & Absent & Absent & Present & $\leq 5.0$ & $\leq 50$ & Hemi & 2 & 4 & Moderate \\
\hline $3.1-5.0$ & $\leq 37$ & $<35$ & $\leq 300$ & Absent & Absent & Absent & Absent & $\leq 5.0$ & $\leq 50$ & Any & 1 & 4 & Moderate \\
\hline $2.1-3.0$ & $>37$ & $\geq 35$ & $\leq 300$ & Any & Absent & Absent & Absent & $>5.0$ & $\leq 50$ & Hemi & 1 & 5 & Moderate \\
\hline $3.1-5.0$ & $>37$ & $\geq 35$ & $\leq 300$ & Absent & Absent & Absent & Absent & $\leq 5.0$ & $\leq 50$ & Minor & 1 & 5 & Moderate \\
\hline$>5.0$ & $\leq 37$ & $\geq 35$ & $<100$ & Any & Absent & Present & Absent & $\leq 5.0$ & $\leq 50$ & Minor & 1 & 5 & Moderate \\
\hline $2.1-3.0$ & $>37$ & $\geq 35$ & $\leq 300$ & Absent & Present & Absent & Absent & $<2.5$ & $\leq 50$ & Minor & 1 & 6 & High \\
\hline $3.1-5.0$ & $\leq 37$ & $\geq 35$ & $>300$ & Absent & Present & Absent & Absent & $>5.0$ & $\leq 50$ & Hemi & 1 & 6 & High \\
\hline$>5.0$ & $\leq 37$ & $\geq 35$ & $\leq 300$ & Absent & Absent & Absent & Absent & $<2.5$ & $\leq 50$ & Extended & $\geq 3$ & 6 & High \\
\hline $3.1-5.0$ & $>37$ & $<35$ & $>300$ & Absent & Present & Present & Absent & $\leq 5.0$ & $\leq 50$ & Minor & 1 & 7 & High \\
\hline$>5.0$ & $>37$ & $<35$ & $>300$ & Absent & Present & Present & Present & $\leq 5.0$ & $>50$ & Extended & $\geq 3$ & 7 & High \\
\hline
\end{tabular}

CCLRS, combined Cox \& logistic ranking system; CA19-9, carbohydrate antigen 19-9; HBV, hepatitis B virus; CEA, carcinoembryonic antigen; AFP, alpha fetoprotein. 
Table S5 Characteristics of the validation cohorts according to the CCLRS ranks

\begin{tabular}{|c|c|c|c|c|c|c|c|c|}
\hline Characteristics & Rank 1 (n=36) & Rank $2(n=40)$ & Rank $3(n=19)$ & Rank $4(n=40)$ & Rank $5(\mathrm{n}=22)$ & Rank $6(n=117)$ & Rank 7 (n=58) & $P$ \\
\hline Validation cohort 1 & $8(22 \%)$ & $4(10 \%)$ & $2(11 \%)$ & $12(29 \%)$ & $7(32 \%)$ & $33(28 \%)$ & $18(31 \%)$ & \\
\hline Validation cohort 2 & 0 & $3(8 \%)$ & 0 & $2(5 \%)$ & $3(14 \%)$ & $2(2 \%)$ & $4(7 \%)$ & \\
\hline Validation cohort 3 & $28(78 \%)$ & $33(83 \%)$ & $17(89 \%)$ & 27 (66\%) & $12(55 \%)$ & $81(69 \%)$ & $36(62 \%)$ & \\
\hline Diabetes & 0 & $1(3 \%)$ & $1(5 \%)$ & $3(7 \%)$ & $5(23 \%)$ & 15 (13\%) & $11(19 \%)$ & 0.013 \\
\hline Cholelithiasis & 0 & $1(3 \%)$ & 0 & $1(2 \%)$ & $5(23 \%)$ & $14(12 \%)$ & $14(24 \%)$ & $<0.001$ \\
\hline Albumin $<35 \mathrm{~g} / \mathrm{L}$ & 0 & 0 & 0 & $2(5 \%)$ & $1(5 \%)$ & $10(9 \%)$ & $15(26 \%)$ & $<0.001$ \\
\hline Platelet count, $\times 10^{9} / \mathrm{L}$ & & & & & & & & 0.001 \\
\hline$<100$ & $10(28 \%)$ & $2(5 \%)$ & $1(5 \%)$ & $4(10 \%)$ & $3(14 \%)$ & $6(5 \%)$ & $1(2 \%)$ & \\
\hline $100-300$ & $24(67 \%)$ & 37 (93\%) & 18 (95\%) & $36(88 \%)$ & $18(82 \%)$ & 99 (85\%) & 49 (84\%) & \\
\hline$>300$ & $2(6 \%)$ & $1(3 \%)$ & 0 & $1(2 \%)$ & $1(5 \%)$ & $11(9 \%)$ & $8(14 \%)$ & \\
\hline HBV infection & $12(33 \%)$ & $8(20 \%)$ & $1(5 \%)$ & $12(29 \%)$ & $7(32 \%)$ & $19(16 \%)$ & $9(16 \%)$ & 0.059 \\
\hline AFP $>50 \mathrm{ng} / \mathrm{mL}$ & $2(6 \%)$ & $1(3 \%)$ & 0 & $5(12 \%)$ & $3(14 \%)$ & $17(15 \%)$ & 17 (29\%) & 0.001 \\
\hline CA19-9 >37 kU/L & 0 & $3(8 \%)$ & 0 & $11(27 \%)$ & $4(18 \%)$ & 92 (79\%) & $58(100 \%)$ & $<0.001$ \\
\hline CEA, ng/mL & & & & & & & & $<0.001$ \\
\hline$<2.5$ & $28(78 \%)$ & $23(58 \%)$ & $19(100 \%)$ & 20 (49\%) & $12(55 \%)$ & $49(42 \%)$ & $9(16 \%)$ & \\
\hline $2.5-5.0$ & $6(17 \%)$ & $15(38 \%)$ & 0 & $15(37 \%)$ & $7(32 \%)$ & $29(25 \%)$ & $6(10 \%)$ & \\
\hline$>5.0$ & $2(6 \%)$ & $2(5 \%)$ & 0 & $6(15 \%)$ & $3(14 \%)$ & $38(32 \%)$ & $43(74 \%)$ & \\
\hline \multicolumn{9}{|l|}{ Tumor size } \\
\hline$\leq 2.0$ & $17(47 \%)$ & $3(8 \%)$ & $2(11 \%)$ & $3(7 \%)$ & $3(14 \%)$ & $3(3 \%)$ & 0 & $<0.001$ \\
\hline $2.0-3.0$ & $16(44 \%)$ & $4(10 \%)$ & 0 & $11(27 \%)$ & $3(14 \%)$ & $14(12 \%)$ & 0 & \\
\hline $3.1-5.0$ & $3(8 \%)$ & 17 (43\%) & 17 (89\%) & $8(20 \%)$ & $6(27 \%)$ & 34 (29\%) & $6(10 \%)$ & \\
\hline$>5$ & 0 & $16(40 \%)$ & 0 & $19(46 \%)$ & $10(45 \%)$ & $65(56 \%)$ & $52(90 \%)$ & \\
\hline Tumor number & & & & & & & & $<0.001$ \\
\hline 1 & 36 (100\%) & 39 (98\%) & $19(100 \%)$ & $33(80 \%)$ & 17 (77\%) & 89 (76\%) & $29(50 \%)$ & \\
\hline 2 & 0 & 0 & 0 & $2(5 \%)$ & $2(9 \%)$ & $7(6 \%)$ & $6(10 \%)$ & \\
\hline$\geq 1$ & 0 & $1(3 \%)$ & 0 & $6(15 \%)$ & $3(14 \%)$ & $20(17 \%)$ & $23(40 \%)$ & \\
\hline Resection type & & & & & & & & 0.001 \\
\hline Minor & $31(86 \%)$ & 27 (68\%) & 17 (89\%) & $23(56 \%)$ & $10(45 \%)$ & 66 (56\%) & $26(45 \%)$ & \\
\hline Hemi & $5(14 \%)$ & $12(30 \%)$ & $1(5 \%)$ & $16(39 \%)$ & $8(36 \%)$ & $44(38 \%)$ & 27 (47\%) & \\
\hline Extended & 0 & $1(3 \%)$ & $1(5 \%)$ & $2(5 \%)$ & $4(18 \%)$ & $6(5 \%)$ & $5(9 \%)$ & \\
\hline Lymph node metastasis & 0 & $1(3 \%)$ & $1(5 \%)$ & $2(5 \%)$ & $9(41 \%)$ & 41 (35\%) & $43(74 \%)$ & $<0.001$ \\
\hline
\end{tabular}

Data are $\mathrm{n}(\%)$. The validation cohort 1 was drawn from Renji Hospital (School of Medicine, Shanghai Jiao Tong University), validation cohort 2 from Mengchao Hepatobiliary Hospital (Fujian Medical University), and validation cohort 3 from Zhongshan Hospital (Fudan University). Characteristics were compared using the Pearson's chi-square test. CCLRS, combined Cox \& logistic ranking system; HBV, hepatitis B virus; AFP, alpha fetoprotein; CA19-9, carbohydrate antigen 19-9; CEA, carcinoembryonic antigen. 

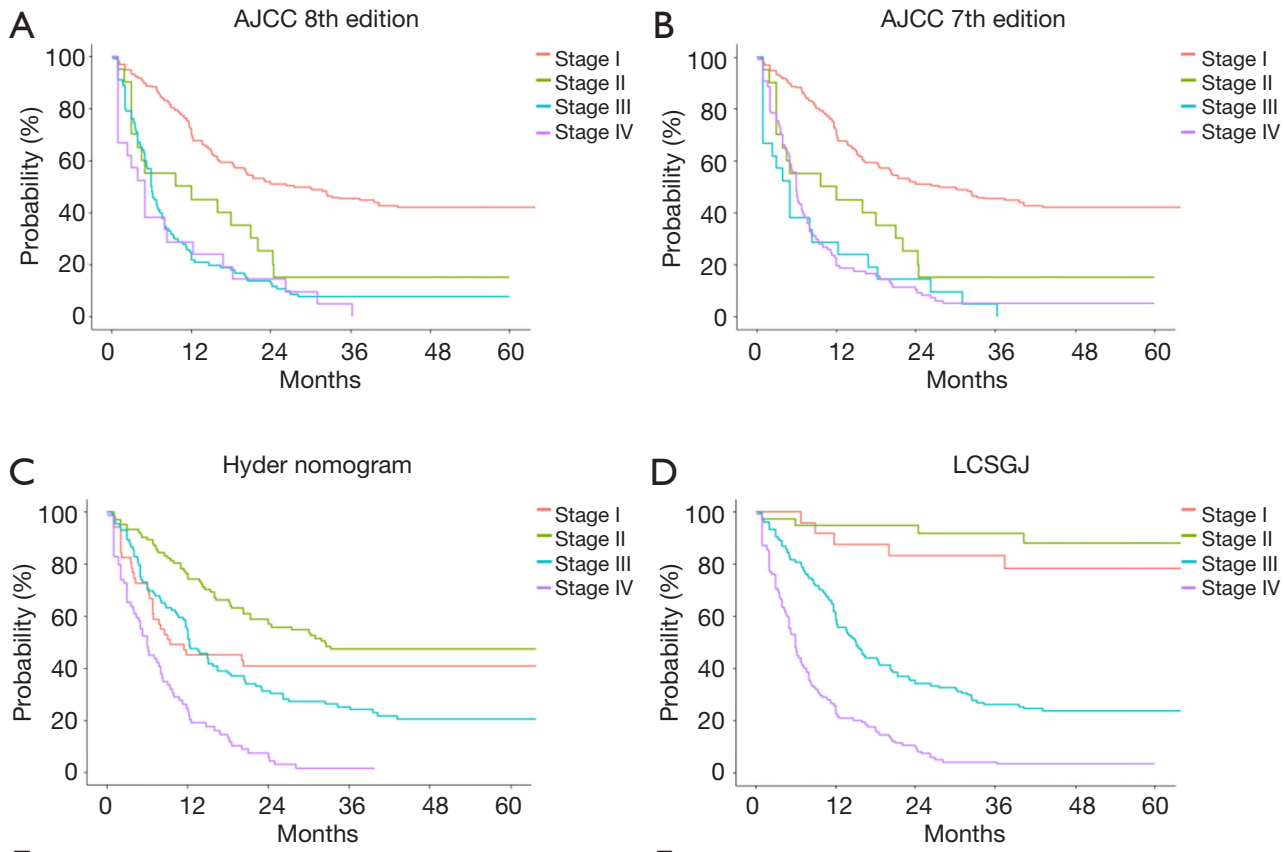

D LCSGJ
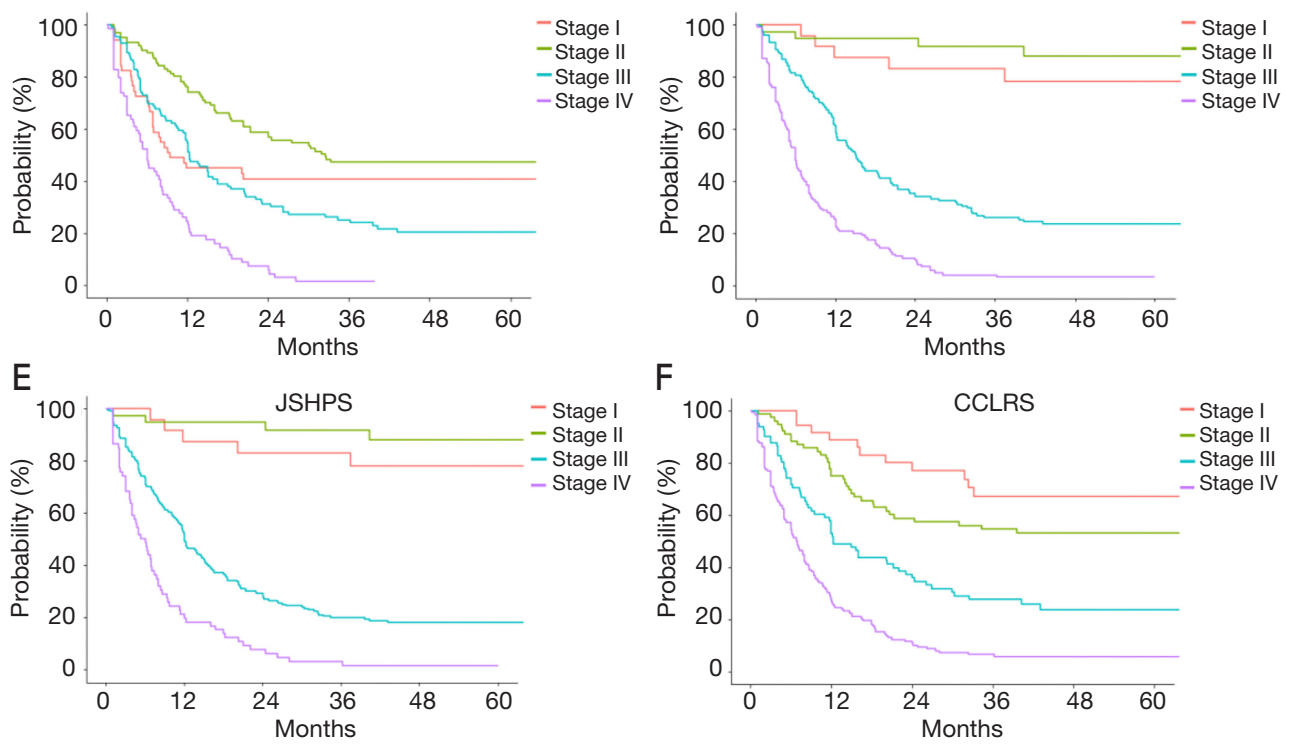

Figure S3 Kaplan-Meier estimations comparing the CCLRS and preexisting ICC staging systems. (A) American Joint Committee on Cancer (AJCC) eighth edition. (B) AJCC seventh edition. (C) Hyder nomogram. (D) Liver Cancer Study Group of Japan (LCSGJ). (E) Japanese Society of Hepato-Biliary Pancreatic Surgery (JSHBPS). (F) CCLRS. To compare with other preexisting systems with 4 stages, the patients in validation cohorts were divided into 4 groups according to the interquartile of risk probability (stage I, 0 to Q1; stage II, Q1 to Q2; stage III, Q2 to Q3; stage IV, Q3 to 1). CCLRS, combined Cox \& logistic ranking system; ICC, intrahepatic cholangiocarcinoma. 

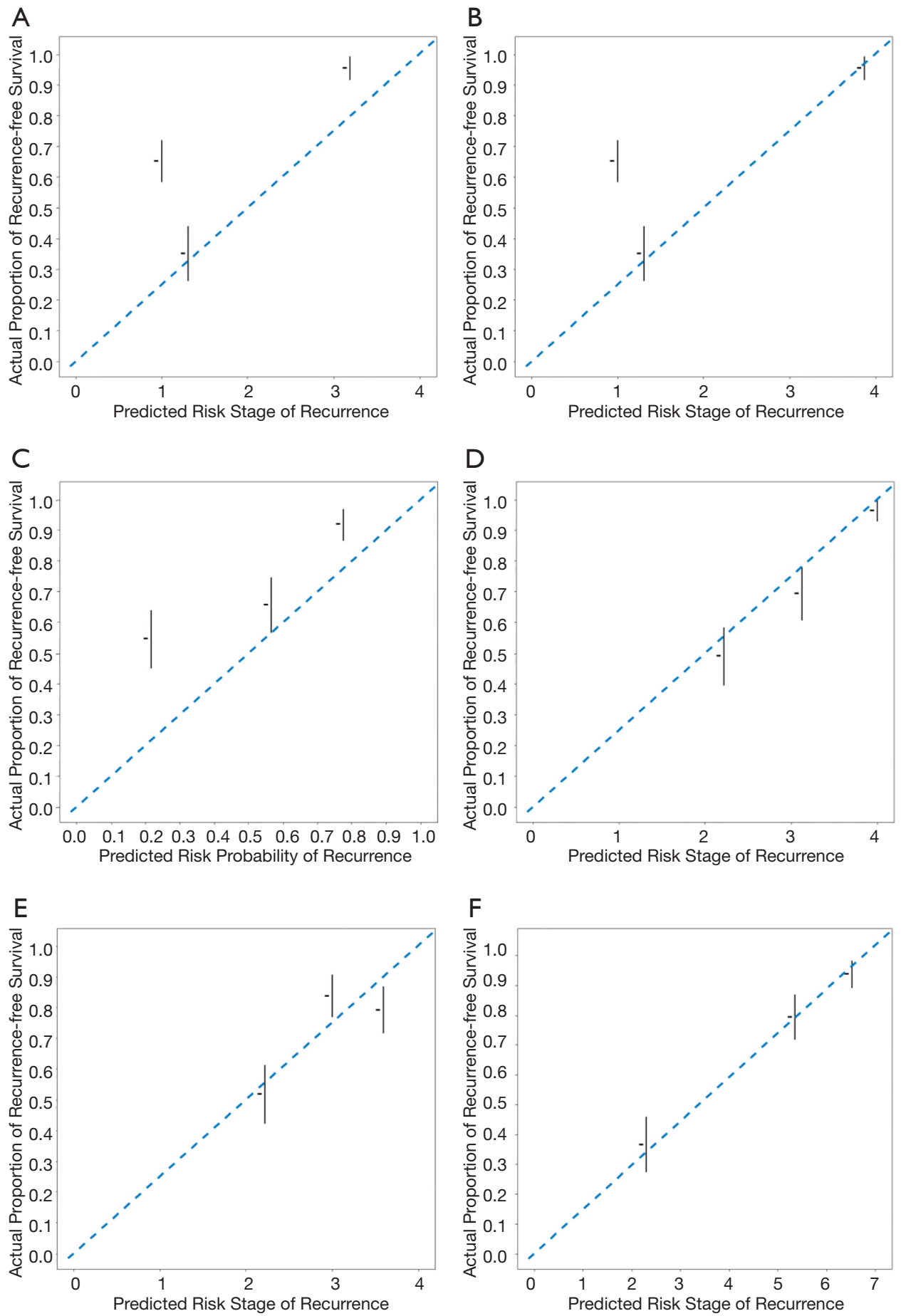

Figure S4 Calibration plots for CCLRS and other ICC prognostic prediction models. (A) American Joint Committee on Cancer (AJCC) eighth edition. (B) AJCC seventh edition. (C) Hyder nomogram. (D) Liver Cancer Study Group of Japan (LCSGJ). (E) Japanese Society of Hepato-Biliary Pancreatic Surgery (JSHBPS). (F) CCLRS. CCLRS, combined Cox \& logistic ranking system. 